\title{
Model-based satellite image fusion
}

Aanæs, Henrik; Sveinsson, J. R.; Nielsen, Allan Aasbjerg; Bøvith, Thomas; Benediktsson, J. A.

Published in:

IEEE Transactions on Geoscience and Remote Sensing

Link to article, DOI:

10.1109/TGRS.2008.916475

Publication date:

2008

Document Version

Publisher's PDF, also known as Version of record

Link back to DTU Orbit

Citation (APA):

Aanæs, H., Sveinsson, J. R., Nielsen, A. A., Bøvith, T., \& Benediktsson, J. A. (2008). Model-based satellite image fusion. IEEE Transactions on Geoscience and Remote Sensing, 46(5), 1336-1346.

https://doi.org/10.1109/TGRS.2008.916475

\section{General rights}

Copyright and moral rights for the publications made accessible in the public portal are retained by the authors and/or other copyright owners and it is a condition of accessing publications that users recognise and abide by the legal requirements associated with these rights.

- Users may download and print one copy of any publication from the public portal for the purpose of private study or research.

- You may not further distribute the material or use it for any profit-making activity or commercial gain

- You may freely distribute the URL identifying the publication in the public portal

If you believe that this document breaches copyright please contact us providing details, and we will remove access to the work immediately and investigate your claim. 


\title{
Model-Based Satellite Image Fusion
}

\author{
Henrik Aanæs, Johannes R. Sveinsson, Senior Member, IEEE, Allan Aasbjerg Nielsen, \\ Thomas Bøvith, and Jón Atli Benediktsson, Fellow, IEEE
}

\begin{abstract}
A method is proposed for pixel-level satellite image fusion derived directly from a model of the imaging sensor. By design, the proposed method is spectrally consistent. It is argued that the proposed method needs regularization, as is the case for any method for this problem. A framework for pixel neighborhood regularization is presented. This framework enables the formulation of the regularization in a way that corresponds well with our prior assumptions of the image data. The proposed method is validated and compared with other approaches on several data sets. Lastly, the intensity-hue-saturation method is revisited in order to gain additional insight of what implications the spectral consistency has for an image fusion method.
\end{abstract}

Index Terms - Generative model, image fusion, satellite images, sensor fusion, spectral consistency.

\section{INTRODUCTION}

$\mathbf{S}$ ATELLITES provide very valuable data about the Earth, e.g., for environmental monitoring, weather forecasting, map making, and military intelligence. Satellites are expensive both to build and operate. This implies that we are somehow obliged to do the best with the data we get from existing satellites, e.g., by combining the output from different sensors and to increase the knowledge we can infer. In this paper, we deal with the merging of low spatial and high spectral resolution satellite images with high spatial and low spectral ones with the aim of creating high spatial and high spectral images. This is also known as image fusion, which is a subset of sensor or data fusion.

Image fusion can be done at several levels: pixel, feature, object, and decision levels, depending on the intended use of the fused image. In this paper, we are only concerned about pixellevel fusion, and when the terms "image fusion" or "fusion" are used, pixel-level fusion is intended. In the current context, fusion is the next step after preprocessing and the step before further processing, such as segmentation and classification.

As argued hereafter, satellite image fusion is an ill-posed inverse problem, implying that we do not have enough informa-

Manuscript received May 31, 2007; revised October 30, 2007. This work was supported in part by the Research Fund of the University of Iceland.

H. Aanæs is with the Department of Informatics and Mathematical Modelling, Technical University of Denmark, 2800 Lyngby, Denmark.

J. R. Sveinsson and J. A. Benediktsson are with the Department of Electrical and Computer Engineering, University of Iceland, 101 Reykjavík, Iceland.

A. A. Nielsen is with the National Space Institute, Technical University of Denmark, 2800 Lyngby, Denmark.

T. Bøvith is with the National Space Institute, Technical University of Denmark, 2800 Lyngby, Denmark, and also with the Danish Meteorological Institute, 2100 Copenhagen, Denmark.

Digital Object Identifier 10.1109/TGRS.2008.916475 tion to solve the problem. Thus, in a sense, we have to "make up" the missing pieces of information, which, in practice, implies the use of prior knowledge either implicitly or explicitly. To address this problem, the approach taken in this paper is to consider the generative models of the data, i.e., models of how the images are formed. This, in turn, allows us to distinguish between exactly what we do know and what we do not know regarding the desired fused image. This has the advantage that the proposed method uses the available information and we can deliberately construct prior models dealing with what we do not know.

\section{A. Relation to Other Works}

During the past two decades, several fusion techniques have been proposed. Most of these techniques are based on the compromise between the desired spatial enhancement and the spectral consistency. The intensity-hue-saturation (IHS) method [1]-[4] has been widely used for red, green, and blue (RGB) color-composite fusion. The IHS is probably the most popular image fusion approach because of its low computational cost. The Brovey [5] sharpening method is a simple technique, and it can be performed on individual bands. Principal-componentanalysis [6] and wavelet-based fusion methods [7]-[10] have also been proposed. All these methods fall in the category of component-substitution methods. All of them can cause color distortion if the spectral range of the intensity replacement image is different from the spectral range covered by the bands used in a color composite. That is to say, the fused images obtained by these methods have high spatial quality, but they suffer from spectral distortions. To achieve an image with high fidelity to the original spectral information, the smoothing filterbased intensity modulation fusion technique was proposed by Liu [8]. However, the images obtained by using that method are blurred compared with other fusion techniques. It was observed that this blurring was a direct result of inaccurate coregistration between the up-scaled multispectral and captured panchromatic images. A good introduction to satellite image fusion can be found in [11].

In this paper, a novel multisensor image fusion technique is presented. The method is model based, and the fused images obtained spectrally are consistent by design. The problem of satellite image fusion is an ill-posed problem, and therefore, regularization is necessary for its solution. For this purpose, the framework for pixel neighborhood regularization is presented in this paper. Using this framework, we can do the regularization consciously and also formulate it in a way that corresponds well with our prior assumptions on the image data. 


\section{B. Notation and Paper Outline}

The scope of this paper is to estimate a high-resolution multichannel image from both low-resolution multichannel and high-resolution panchromatic images. The presented theory and methodology are valid for an arbitrary number of channels and an arbitrary ratio between the spatial resolution of the lowand high-resolution images. For ease of presentation and without loss of generality, it is assumed (if nothing else is stated) that, in this paper, we work with three channels (red, green, and blue) and the ratio between high and low spatial resolution pixels is one to four by four, i.e., $1: 16$. The notation used in this paper is as follows: We want to estimate $\left(\mathbf{R}_{i j}^{\text {high }}, \mathbf{G}_{i j}^{\text {high }}, \mathbf{B}_{i j}^{\text {high }}\right)$ from a low-resolution RGB image $\left(\mathbf{R}_{i}^{\text {low }}, \mathbf{G}_{i}^{\text {low }}, \mathbf{B}_{i}^{\text {low }}\right)$ and a high-resolution panchromatic image $\mathbf{P}_{i j}^{\text {high }}$. The indices are such that the first one $i$ enumerates the low-resolution pixels and the second one $j \in\{1, \ldots, 16\}$ enumerates the associated high-resolution pixels.

The outline of this paper is as follows. In Section II, it is argued that the satellite image fusion is inherently ill posed. In Section III, this is followed by the introduction of our sensor or observation model with the constraints that we draw from it. An initial solution applying these constraints is given in Section IV. Section V presents additional ways of regularizing the solution by neighborhood comparison. In Section VI, the IHS method is set in the context of this paper, giving additional insight. An experimental comparison of our proposed method and a few selected other approaches from the literature are reported in Section VII. This is followed by a conclusion of this paper and an Appendix dealing with computational issues relating to the proposed method.

\section{ILL-POSED PROBLEM}

As mentioned earlier, we argue that any satellite image fusion method needs regularization. This is due to the image fusion problem being ill posed or under constraint, which can be seen by simple variable counting. That is, for each low-resolutionimage pixel $i$, we have three values from the low-resolution pixel $\left(\mathbf{R}_{i}^{\text {low }}, \mathbf{G}_{i}^{\text {low }}, \mathbf{B}_{i}^{\text {low }}\right)$ and 16 panchromatic values, i.e., $19(=16+3)$ in all. On the other hand, we need to estimate 16 RGB values, i.e., $48(=16 \cdot 3)$, which give us a shortage of $29(=48-19)$ values. Because no other information is available, we have to produce these 29 dimensions or values by regularization, preferably by modeling our prior assumption of the data. This regularization can either be explicit or implicit. For example, in the IHS method, regularization is implicitly done by assuming that the hue and saturation values of all highresolution pixels are equal to the low-resolution pixel. That is not necessarily the right solution, and probably, it is not.

\section{OBSERVATION MODEL}

The methods presented here take their outset in an "assumed" observation model for the satellite image sensor, which is also seen as a generative model of the data.

This observation model, which is based on the response of a given pixel and a data channel, is given by the frequency composition and intensity of the incoming light $L(\mathbf{x}, \omega)$, where $\mathbf{x}$ and $\omega$ denote position and frequency, respectively, and the spectral response function for that channel $F(\omega),{ }^{1}$ cf., Fig. 1. This response can be expressed as

$$
\int_{A} \int_{\Omega} L(\mathbf{x}, \omega) F(\omega) d \omega d \mathbf{x}
$$

the frequency runs over a relevant spectral range $\Omega$. The integral (1) runs over the area $A$ corresponding to the sensor area related to the pixel.

\section{A. Inner Product Space}

The response in (1) is a convolution between the spectrum of the incoming light $L(\mathbf{x}, \omega)$ and the response function of the channels $F(\omega)$. This induces an inner product, e.g., ${ }^{2}$

$$
\left\langle F^{\mathrm{red}}, F^{\mathrm{pan}}\right\rangle=\int_{\Omega} F^{\mathrm{red}}(\omega) F^{\mathrm{pan}}(\omega) d \omega
$$

corresponding to the $\mathcal{L}^{2}$ norm ${ }^{3}$ [13] which introduces a measure of angle and distance. In using this, we are only interested in the observable $4-\mathrm{D}$ subspace spanned by the $\mathbf{R}, \mathbf{G}, \mathbf{B}$, and $\mathbf{P}$ channels. Therefore, as an example, a measure of how much information about $\mathbf{G}^{\text {high }}$ is contained in $\mathbf{P}^{\text {high }}$ is given by ${ }^{4}$

$$
\alpha(G, P)=\frac{\left\langle F^{\text {green }}, F^{\text {pan }}\right\rangle}{\sqrt{\left\langle F^{\text {green }}, F^{\text {green }}\right\rangle\left\langle F^{\text {pan }}, F^{\text {pan }}\right\rangle}}
$$

which is equal to the cosine of the angle between the two spectra. Collecting these normalized inner products between all channels gives

$$
\Sigma=\left[\begin{array}{llll}
\alpha(R, R) & \alpha(R, G) & \alpha(R, B) & \alpha(R, P) \\
\alpha(G, R) & \alpha(G, G) & \alpha(G, B) & \alpha(G, P) \\
\alpha(B, R) & \alpha(B, G) & \alpha(B, B) & \alpha(B, P) \\
\alpha(P, R) & \alpha(P, G) & \alpha(P, B) & \alpha(P, P)
\end{array}\right]
$$

which, in some sense, describes the information between the different channels. In Section IV, this matrix will be interpreted as a correlation matrix, for ease of computation, without us claiming that the spectra are normally distributed.

\section{B. Spectral Consistency}

Assuming that the low- and high-resolution pixels are perfectly aligned, i.e., the areas associated with the different pixels

\footnotetext{
${ }^{1} F(\omega)$ is usually supplied by the sensor manufacturer.

${ }^{2}$ The response functions are independent of position. Therefore, there is no need to integrate over area.

${ }^{3}$ This is an extension of the regular two-norm to an infinite dimensional space. Later, we will just refer to this as the two-norm.

${ }^{4}$ We assume that there is an individual linear stretch of each channel, such that it best fits the dynamic range. Thus, we normalize this criterion to account for this.
} 


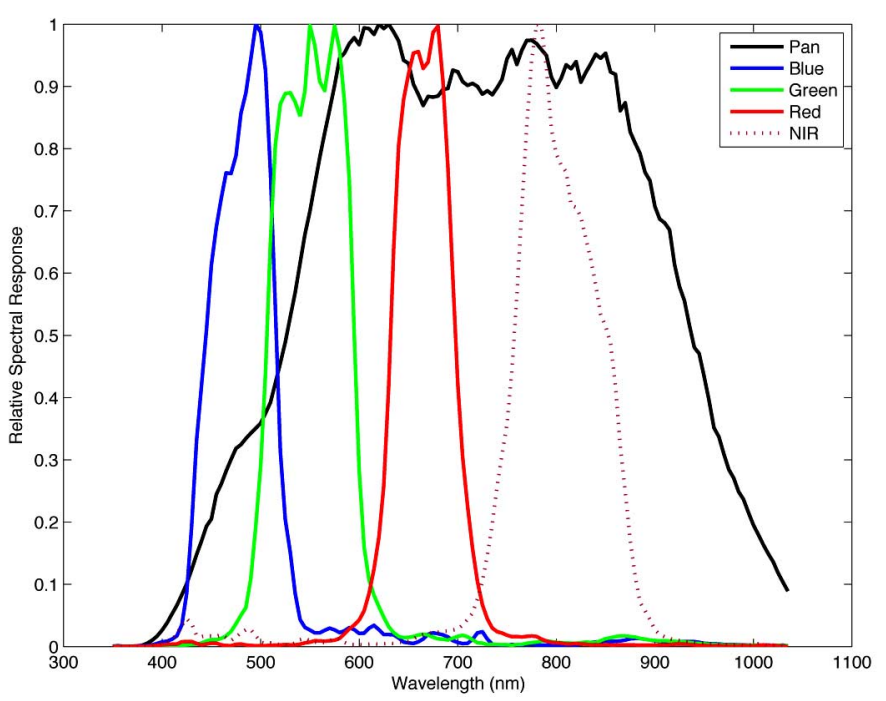

Fig. 1. Spectral response of the IKONOS spectral bands in [12].

satisfy (see Fig. 2)

$$
A_{i}=\bigcup_{j} A_{i j} \quad \text { and } \quad \emptyset=\bigcap_{j} A_{i j} .
$$

Another property derived from (1) is that of spectral consistency. This property is very important for satellite image fusion because spectral distortion may result in unreliable interpretation for applications. By considering any channel, e.g., the blue, spectral consistency can be derived as follows:

$$
\begin{aligned}
\mathbf{B}_{i}^{\text {low }} & =\int_{A_{i}} \int_{\Omega} L(\mathbf{x}, \omega) F(\omega) d \omega d \mathbf{x} \\
& =\sum_{j=1}^{16} \int_{A_{i j}} \int_{\Omega} L(\mathbf{x}, \omega) F(\omega) d \omega d \mathbf{x} \\
& =\sum_{j=1}^{16} \mathbf{B}_{i j}^{\text {high }} .
\end{aligned}
$$

Therefore, the spectral consistency constraint states that ${ }^{5}$

$$
\left[\begin{array}{l}
\mathbf{R}_{i}^{\text {low }} \\
\mathbf{G}_{i}^{\text {low }} \\
\mathbf{B}_{i}^{\text {low }}
\end{array}\right]=\frac{1}{16} \sum_{j=1}^{16}\left[\begin{array}{l}
\mathbf{R}_{i j}^{\text {high }} \\
\mathbf{G}_{i j}^{\text {high }} \\
\mathbf{B}_{i j}^{\text {high }}
\end{array}\right] .
$$

\section{INITIAL SOLUTION}

Deriving an image fusion method from the aforementioned observation model is hindered by $\left(\mathbf{R}^{\text {high }}, \mathbf{G}^{\text {high }}, \mathbf{B}^{\text {high }}\right)$, not being fully observable from the data, as argued in Section II. Therefore, regularization is required. The same is also illustrated by the spectral response functions of the sensor, cf., Fig. 1. Here, it is seen that there are several compositions of incoming light, giving the same response on the panchromatic
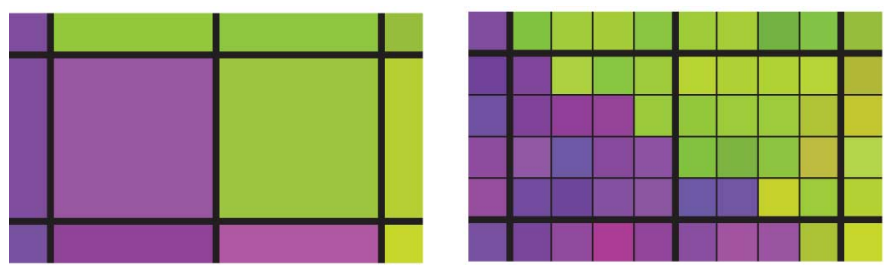

Fig. 2. Here, it is illustrated that, because the same light is supposedly hitting the low and high spatial resolution sensors, the color of the low-resolution pixel is equal to the average of the corresponding high-resolution pixels, as expressed in (5).

channel but highly different on, e.g., the red channel. Thus, $\mathbf{P}^{\text {high }}$ and (5) do not give enough information to fully determine the individual high-resolution multispectral components, e.g., $\mathbf{R}^{\text {high }}$.

Here, it is assumed that the "unknown" part ${ }^{6}$ of the signal is small. Therefore, we regularize the solution by penalizing it with the two-norm, because this is the norm induced by the inner product. This implicitly corresponds to casting the problem in a statistical setting, assuming that an "observation" in $\left(\mathbf{R}^{\text {high }}, \mathbf{G}^{\text {high }}, \mathbf{B}^{\text {high }}, \mathbf{P}^{\text {high }}\right)$ space is normally distributed with covariance $\Sigma$ (4). Thus, the conditional means method [14] is used to solve the problem. Due to the spectral consistency, we know that the mean of $\left(\mathbf{R}_{i j}^{\text {high }}, \mathbf{G}_{i j}^{\text {high }}, \mathbf{B}_{i j}^{\text {high }}\right)$ is equal to $\left(\mathbf{R}_{i}^{\text {low }}, \mathbf{G}_{i}^{\text {low }}, \mathbf{B}_{i}^{\text {low }}\right)$. This gives the following solution to the fusion problem:

$$
\left[\begin{array}{l}
\widehat{\mathbf{R}_{i j}^{\text {high }}} \\
\mathbf{G}_{i j}^{\text {high }} \\
\mathbf{B}_{i j}^{\text {high }}
\end{array}\right]=\widehat{\mathbf{F}}_{i j}=\left[\begin{array}{l}
\mathbf{R}_{i}^{\text {low }} \\
\mathbf{G}_{i}^{\text {low }} \\
\mathbf{B}_{i}^{\text {low }}
\end{array}\right]+\left[\begin{array}{c}
\alpha(R, P) \\
\alpha(G, P) \\
\alpha(B, P)
\end{array}\right]\left(\mathbf{P}_{i j}^{\text {high }}-P_{i}^{\mu}\right)
$$

where $P_{i}^{\mu}$ denotes the mean of the $\mathbf{P}_{i j}^{\text {high }}$ w.r.t. the lowresolution pixel $i$. For ease of notation, this solution is denoted by $\widehat{\mathbf{F}}_{i j}$. The results of this proposed method can be seen in Figs. 5-7 (where this solution is referred to as no smoothing). A couple of comments should be made w.r.t. the solution in (6). First, we do not claim that the observations are normally distributed, just that a reasonable regularization corresponds to it. Second, if there is no "overlap" between the spectral responses of the panchromatic and one of the low-resolution channels, then $\alpha(\cdot, P)$ will be zero. This indicates that there is no information between the two channels and, in effect, omits the last term in (6) for the channel in question. Lastly, it is seen that the solution is spectrally consistent, cf., (5), by construction, because

$$
P_{i}^{\mu}=\frac{1}{16} \sum_{j=1}^{16} \mathbf{P}_{i j}^{\mathrm{high}} \Rightarrow \sum_{j=1}^{16}\left(\mathbf{P}_{i j}^{\mathrm{high}}-P_{i}^{\mu}\right)=0 .
$$

\section{SMOOTHING PRiors}

Although the aforementioned initial solution is spectrally consistent, it still leaves something to be desired, particularly because there is a pronounced blocking effect originating from 
the low-resolution pixels, as seen in Figs. 5-7. As argued in Section VI, this is likely to be the best that can be done on a pixelwise basis. In order to improve this, we propose the augmentation of the method with a spatial prior. This spatial prior should express our expectation that the fused image should be homogeneous or piecewise homogeneous, which corresponds to our general expectation of images.

Specifically, by applying a Markov random-field setting [15], [16] to the problem, the (conditional) smoothing of the image can be expressed as penalizing deviations between neighboring pixels. That is, define the errors

$$
\epsilon_{i j k}=\left[\begin{array}{l}
\mathbf{R}_{i j}^{\text {high }} \\
\mathbf{G}_{i j}^{\text {high }} \\
\mathbf{B}_{i j}^{\text {high }}
\end{array}\right]-\left[\begin{array}{c}
\mathbf{R}_{k}^{\text {high }} \\
\mathbf{G}_{k}^{\text {high }} \\
\mathbf{B}_{k}^{\text {high }}
\end{array}\right], \quad k \in \mathcal{N}_{i j}
$$

where $\mathcal{N}_{i j}$ is the four neighborhood of pixel $i j$. We then regularize by adding the term

$$
\sum_{i, j} \sum_{k \in \mathcal{N}_{i j}} \rho\left(\epsilon_{i j k}\right) w_{i j k}
$$

to (6), where the $w_{i j k}$ denotes appropriate weights, and $\rho$ is the error function

$$
\rho\left(\epsilon_{i j k}\right)=\epsilon_{i j k}^{T} \widetilde{\Sigma}^{-1} \epsilon_{i j k} .
$$

Here, $\widetilde{\Sigma}$ is the $R G B$ part of $\Sigma$, cf., (4). This regularizer is seen as a $w_{i j k}$-dependent smoothing of the image. The reason that $\widetilde{\Sigma}$ is included in (8) is that $\rho\left(\epsilon_{i j k}\right)$ should measure differences in incoming light and not be biased by the correlation of the measured channels.

The spatial prior is merged with the solution in (6) by combining (6) and (8), i.e., define the deviation from $\widehat{\mathbf{F}}_{i j}$ by

$$
\mathbf{D}_{i j}=\left(\left[\begin{array}{l}
\mathbf{R}_{i j}^{\text {high }} \\
\mathbf{G}_{i j}^{\text {high }} \\
\mathbf{B}_{i j}^{\text {high }}
\end{array}\right]-\widehat{\mathbf{F}}_{i j}\right)^{\mathrm{T}} \widetilde{\Sigma}^{-1}\left(\left[\begin{array}{c}
\mathbf{R}_{i j}^{\text {high }} \\
\mathbf{G}_{i j}^{\text {high }} \\
\mathbf{B}_{i j}^{\text {high }}
\end{array}\right]-\widehat{\mathbf{F}}_{i j}\right)
$$

then, the combined objective function becomes

$$
\min _{\mathbf{R}^{\text {high }}, \mathbf{G}^{\text {high }}, \mathbf{B}^{\text {high }}} \sum_{i j}\left(\mathbf{D}_{i j}+\gamma \sum_{k \in \mathcal{N}_{i j}} \rho\left(\epsilon_{i j k}\right) w_{i j k}\right)
$$

where $\gamma$ is a tuning parameter encapsulating the weight of the smoothness term that is relative to the data term. Note that this is a least squares (LS) problem in $\mathbf{R}^{\text {high }}, \mathbf{G}^{\text {high }}, \mathbf{B}^{\text {high }}$ and (11) is under the condition that the solution is spectrally consistent. This latter characteristic is dealt with in Section V-A. In the experiments relating to this paper, $\gamma$ was selected in the range of one to five. The value of $\gamma$, however, depends highly on the intrinsic scaling of the two combined terms. Different range for the $\gamma$ may be appropriate in other problems.

The problem is now reduced to setting the appropriate weights $w_{i j k}$ to best capture the prior assumption of the fused image. Usually, these weights are set simultaneously with solving (11), because the smoothed solution is the best clue to where the edges in the image should be, cf., e.g., [15] and [16]. In general, the optimization problem in (11) is thus "big" and nonlinear. This is not the case here because we have the highresolution panchromatic image, which is a very good source of information on where edges should be. Here, the weights $w_{i j k}$ are thus based on the panchromatic image, such that the fused image shall have homogeneous regions where the panchromatic image has them. To illustrate this, we have tried two general approaches, cf., Sections V-B and C. The results with uniform smoothing (all $w_{i j k}=1$ ) are also presented for comparison. The results are given in Section VII. By general approaches, we mean that the two investigated approaches are good typical examples of what might be used; thus, they are not the results of a long investigation of what is the best weighting scheme.

\section{A. Ensuring Spectral Consistency}

Extending (6)-(11) implies that the LS solution is not spectrally consistent, cf., (5), and has to be added as a specific condition to the solution. This is done by noting that (5) defines a 15-D hyperplane in 16 dimensions on which the solution should be located. We can thus reparameterize the solution space such that the only possible values are on this hyperplane. This is accomplished by introducing

$$
\mathcal{R}_{i j}^{\text {high }}, \mathcal{G}_{i j}^{\text {high }}, \mathcal{B}_{i j}^{\text {high }}, \quad j \in\{1, \ldots, 15\}
$$

which map to the pixel of the fused image and variables in (1) as follows:

$$
\begin{aligned}
& {\left[\begin{array}{l}
\mathcal{R}_{i j}^{\text {high }} \\
\mathcal{G}_{i j}^{\text {high }} \\
\mathcal{B}_{i j}^{\text {high }}
\end{array}\right]=\left[\begin{array}{l}
\mathbf{R}_{i j}^{\text {high }} \\
\mathbf{G}_{i j}^{\text {high }} \\
\mathbf{B}_{i j}^{\text {high }}
\end{array}\right], \quad j \in\{1, \ldots, 15\}} \\
& {\left[\begin{array}{l}
\mathcal{R}_{i, 16}^{\text {high }} \\
\mathcal{G}_{i, 16}^{\text {high }} \\
\mathcal{B}_{i, 16}^{\text {high }}
\end{array}\right]=16\left[\begin{array}{l}
\mathbf{R}_{i}^{\text {low }} \\
\mathbf{G}_{i}^{\text {low }} \\
\mathbf{B}_{i}^{\text {low }}
\end{array}\right]-\sum_{j=1}^{15}\left[\begin{array}{l}
\mathcal{R}_{i j}^{\text {high }} \\
\mathcal{G}_{i j}^{\text {high }} \\
\mathcal{B}_{i j}^{\text {high }}
\end{array}\right] .}
\end{aligned}
$$

An important property of this mapping is that it is affine. By plugging (12) into (11), a new objective function in $\mathcal{R}_{i j}^{\text {high }}, \mathcal{G}_{i j}^{\text {high }}, \mathcal{B}_{i j}^{\text {high }}$, which is spectrally consistent by design, and an LS system are obtained. This, in turn, has nice implications for computing a solution (see Appendix). It should be noted that solving (11) without this parameterization (or another expression of the spectral consistency constraint) will, in general, yield nonspectrally consistent solutions. It is noted that this parameterization is intrinsic in the predictor for satellite image fusion presented in [17], where it is also noted that it has the desirable characteristic of spectral consistency.

\section{B. Edge-Induced Weights}

We are, in a sense, trying to transfer edges from the panchromatic image to the fused RGB image. Thus, the first of the 

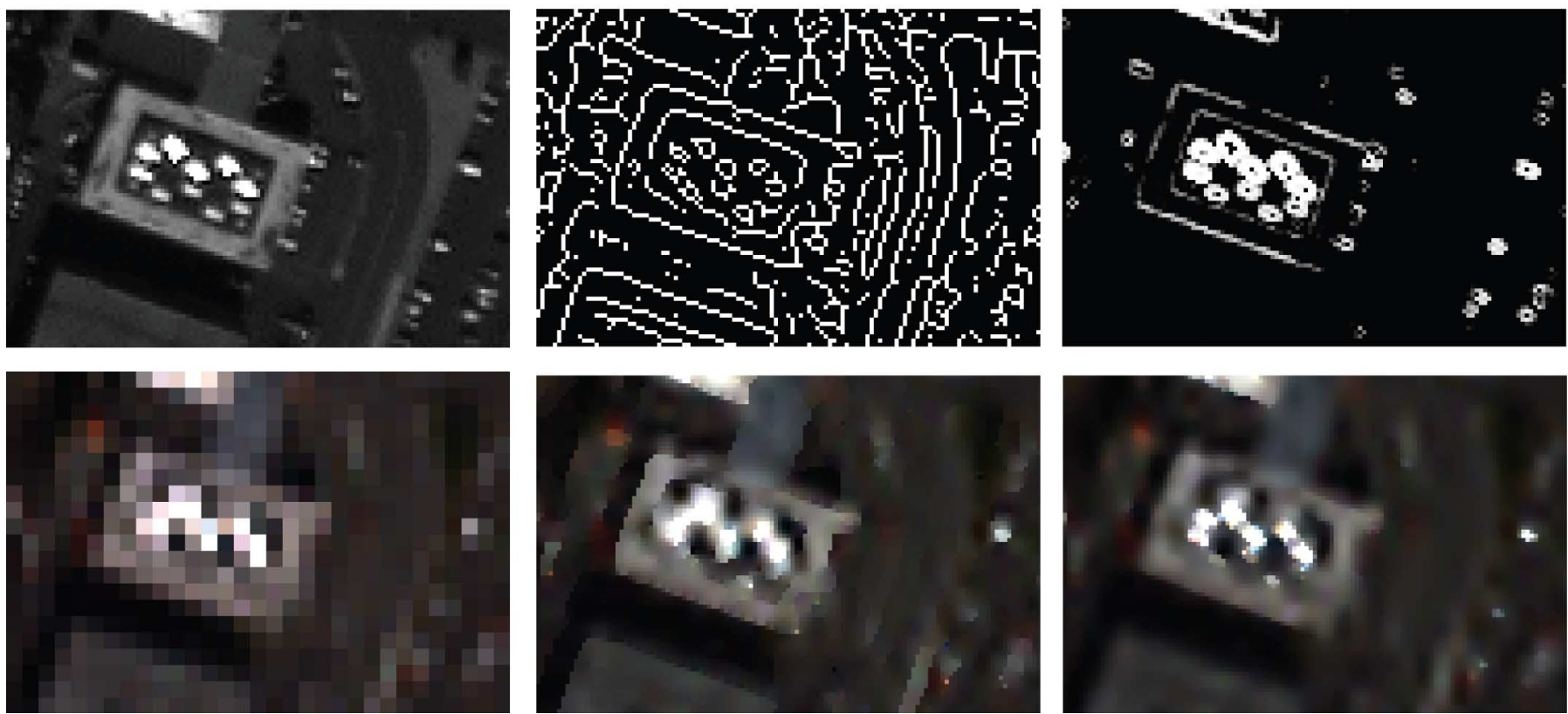

Fig. 3. Sample from satellite image data set. (Top left) Panchromatic image. (Top center) Extracted edges via the Canny edge detector. (Top right) Weight set via (13) with white being zero. Here, a $\lambda$ of 0.05 is used. (Bottom left) Low-resolution multispectral image. (Bottom center) Resulting fused image with weight set according to the Canny weights—-see top center. (Bottom right) Resulting fused image with weight set via (13)—see top right.

general approaches we use for setting the weights in (11) are as follows.

1) Extract edges in the panchromatic image, see Fig. 3, top center.

2) If there is an edge between pixel $i j$ and its $k$ th neighbor, set the weight to zero, or else, set the appropriate weight to one.

The extracted edges are obtained via the Canny edge detector [18]. There are, however, a multitude of methods for edge extraction, many of which could be used successfully, e.g., via the total variation method [19], as used in [20]. The results using the Canny edges are shown in Fig. 3 and Figs. 5-7.

\section{Gradient-Induced Weights}

Setting the weights via edge extraction gives results that are somewhat blocky to sharp edges, as can be seen in Fig. 3 and Figs. 5-7. This is partly due to the weights being either zero or one. Therefore, the algorithm either smooths fully the one between two pixels or does not smooth at all. Thus, it seems natural to let the weights have a continuous transition from zero to one and, by that, introducing the concept of "degree of edginess." That is the basis for our second general weighting scheme.

The simplest form of the second weighting scheme would be letting the weights be proportional to the inverse gradient magnitude of the panchromatic image, i.e.,

$$
w_{i j k} \propto \frac{1}{\left\|\nabla \mathbf{P}_{i j}^{\text {high }}\right\|}
$$

which however did not turn out too successful on our data. Instead, we adapted a method from nonlinear image diffusion

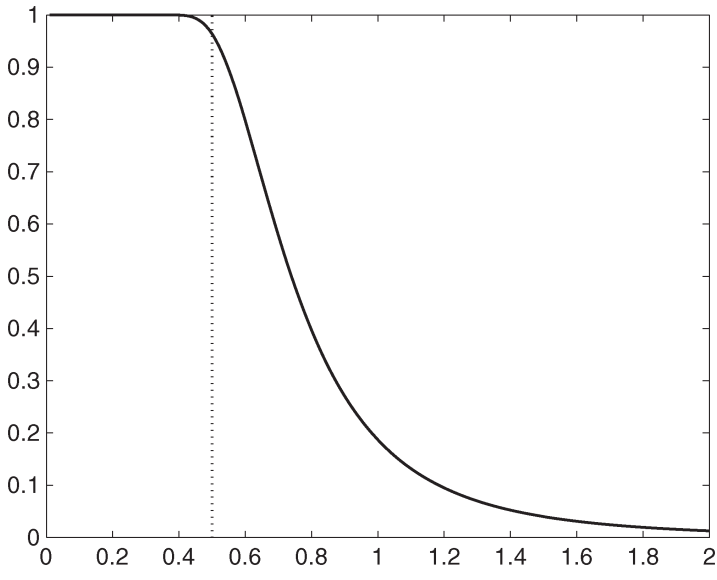

Fig. 4. Function in (13) as a function of the gradient magnitude $\left\|\nabla \mathbf{P}_{i j}^{\text {high }}\right\|$ with $\lambda=0.5$, which is denoted by the dotted line.

[21], which is proposed in [22]. Here, the amount of smoothing is a nonlinear function of the image gradient magnitude-here, the panchromatic image. The function in question is

$$
w_{i j k}=1-\exp \left(\frac{-3.31488}{\left(\frac{\left\|\nabla \mathbf{P}_{i j}^{\text {high }}\right\|}{\lambda}\right)^{4}}\right)
$$

where $\lambda$ is a steering parameter, indicating how large the gradient should be in order to be an edge, see Fig. 4. The results of this scheme can be seen in Fig. 3 and Figs. 5-7. A note on (13): This function, including the -3.31488 , has proven successful in the nonlinear diffusion literature; for more on this matter, see [21]. 

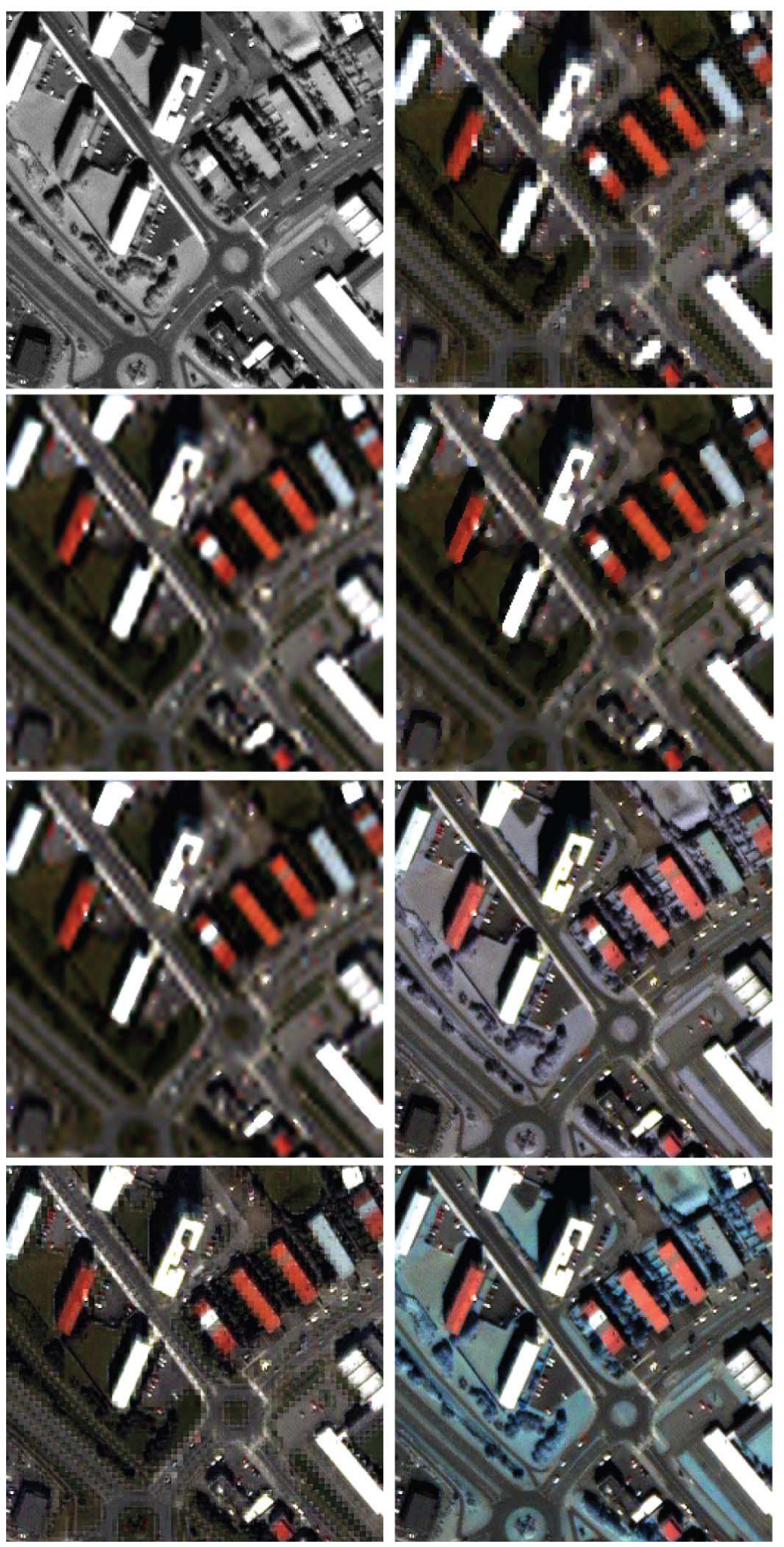

Fig. 5. Results of the image fusion strategies. A sample of the resulting images of the IKONOS data set. (Top left) Input panchromatic image. (Top right) No smoothing. (Row 2 left) Uniform weights. (Row 2 right) Edgeinduced weighting. (Row 3 left) Gradient-induced weighting. (Row 3 right) IHS method. (Bottom left) Mean-corrected IHS method. (Bottom right) Brovey method. The images have been stretched for better visualization.

\section{NOTE ON THE IHS METHOD}

Probably, the most popular method for image fusion is the IHS method, where the fusion is performed in IHS space by letting the hue and saturation of a fused pixel be equal to the corresponding low-resolution pixel and the intensity $\mathbf{I}_{i j}$ be equal to the panchromatic channel, i.e.,

$$
\mathbf{I}_{i j}=\frac{\mathbf{R}_{i j}^{\mathrm{high}}+\mathbf{G}_{i j}^{\mathrm{high}}+\mathbf{B}_{i j}^{\mathrm{high}}}{3}=\mathbf{P}_{i j}^{\mathrm{high}} .
$$
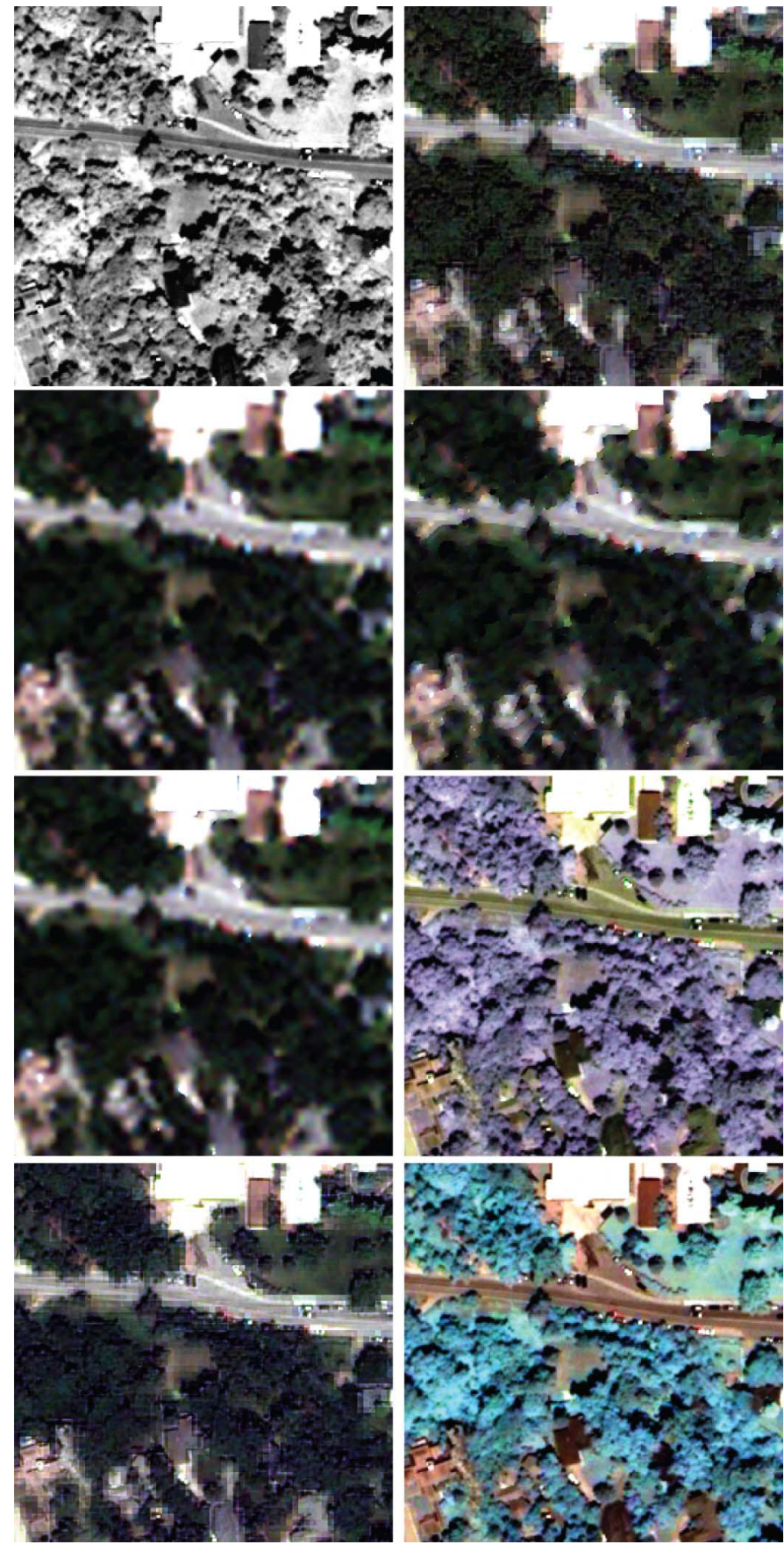

Fig. 6. Results of the image fusion strategies. A sample of the resulting images of the QuickBird data set. (Top left) Input panchromatic image. (Top right) No smoothing. (Row 2 left) Uniform weights. (Row 2 right) Edgeinduced weighting. (Row 3 left) Gradient-induced weighting. (Row 3 right) IHS method. (Bottom left) Mean-corrected IHS method. (Bottom right) Brovey method. The images have been stretched for better visualization.

Combining (5) and (14), it is seen that

$$
\begin{aligned}
\frac{1}{16} \sum_{j=1}^{16} \mathbf{P}_{i j}^{\text {high }} & =\frac{1}{16} \sum_{j=1}^{16} \frac{\mathbf{R}_{i j}^{\text {high }}+\mathbf{G}_{i j}^{\text {high }}+\mathbf{B}_{i j}^{\text {high }}}{3} \\
& =\frac{\mathbf{R}_{i}^{\text {low }}+\mathbf{G}_{i}^{\text {low }}+\mathbf{B}_{i}^{\text {low }}}{3}=\mathbf{I}_{i} .
\end{aligned}
$$




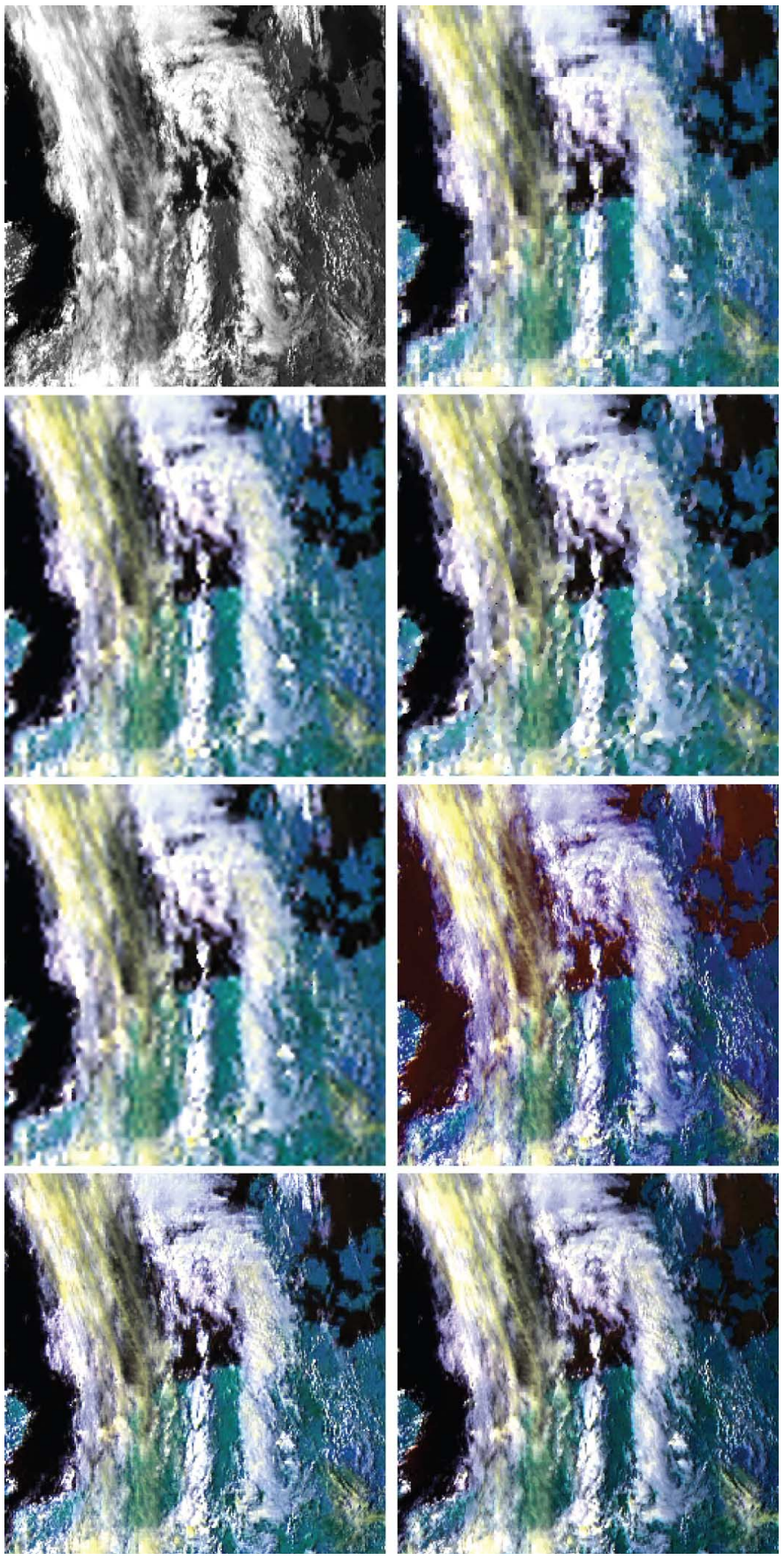

Fig. 7. Results of the image fusion strategies. A sample of the resulting images of the Meteosat data set. (Top left) Input panchromatic image. (Top right) No smoothing. (Row 2 left) Uniform weights. (Row 2 right) Edgeinduced weighting. (Row 3 left) Gradient-induced weighting. (Row 3 right) IHS method. (Bottom left) Mean-corrected IHS method. (Bottom right) Brovey method. The images have been stretched for better visualization.

This implies that if the IHS method should be spectrally consistent, the mean of the panchromatic channel over a low-resolution pixel should equal the corresponding lowresolution intensity. This will generally not be the case, as shown in Fig. 1, where the spectral response function of the panchromatic channel is far from the sum of the red, green, and blue response functions. This fact is also validated experimentally, as reported in Table II.
That leads us to propose a modification of the IHS method, which consists of appropriately correcting the mean of the panchromatic channel. The correction is done by using $\widetilde{\mathbf{P}}_{i j}^{\text {high }}$ instead of $\mathbf{P}_{i j}^{\text {high }}$, where $\widetilde{\mathbf{P}}_{i j}^{\text {high }}$ is defined as follows:

$$
\widetilde{\mathbf{P}}_{i j}^{\text {high }}=\mathbf{P}_{i j}^{\text {high }} \frac{\mathbf{I}_{i}}{\frac{1}{16} \sum_{j=1}^{16} \mathbf{P}_{i j}^{\text {high }}}=\mathbf{I}_{i j} .
$$

It is seen that using $\widetilde{\mathbf{P}}^{\text {high }}$ instead of $\mathbf{P}^{\text {high }}$ in the IHS method gives a spectrally consistent result.

The results of using this mean-corrected IHS method are seen in Figs. 5-7. It is seen that this method, although spectrally consistent, yields a blocky result that is similar to that of our initial solution presented in Section IV. This seems to indicate that in order to get a spectrally consistent result with a simple per pixel method, you need to endure a blocking effect. An explanation is that the discrepancy between the RGB response functions and the panchromatic response function, as seen in Fig. 1, gives a varying mean offset for each block.

This mean-corrected IHS method, in some sense, resembles the Brovey method or transform, cf., [5] and [23], which is thus included for experimental comparison. The Brovey method is given by

$$
\left[\begin{array}{l}
\widehat{\mathbf{R}_{i j}^{\text {high }}} \\
\mathbf{G}_{i j}^{\text {high }} \\
\mathbf{B}_{i j}^{\text {high }}
\end{array}\right]=\left[\begin{array}{l}
\mathbf{R}_{i}^{\text {low }} \\
\mathbf{G}_{i}^{\text {low }} \\
\mathbf{B}_{i}^{\text {low }}
\end{array}\right] \frac{\mathbf{P}_{i j}^{\text {high }}}{\frac{\mathbf{R}_{i}^{\text {low }}+\mathbf{G}_{i}^{\text {low }}+\mathbf{B}_{i}^{\text {low }}}{} .}
$$

It should also be noted that other methods have been proposed for addressing the issue of spectral consistency with the IHS method, cf., e.g., [24].

\section{EXPERIMENTAL COMPARISONS AND DISCUSSION}

To experimentally investigate and compare the proposed methods, we have applied them to three different data sets from three different types of satellites, each depicting a different type of landscape. The proposed methods were compared with the IHS and Brovey methods for comparison. The investigated methods are summarized in Table I. The three data sets are as follows.

1) IKONOS. An image of an urban area is taken by the IKONOS earth imaging satellite [12]. The low-resolution image consists of four bands $\mathbf{R}, \mathbf{G}, \mathbf{B}$, and near infrared (NIR) (in this paper, we only use $\mathbf{R}, \mathbf{G}$, and $\mathbf{B}$ ), and the ratio between the panchromatic and low-resolution images is 16 or four by four.

2) QuickBird. An image of a forest landscape is bisected by roads, and it contained a few large buildings. A city emerges toward the right side. This image is taken by the QuickBird satellite [25]. The low-resolution image consists of four bands $\mathbf{R}, \mathbf{G}, \mathbf{B}$, and NIR (in this paper, we use only $\mathbf{R}, \mathbf{G}$, and $\mathbf{B}$ ), and the ratio between

\footnotetext{
${ }^{7}$ Partly for gaining insight into what spectral consistency implies for satellite image fusion methods.
} 
TABLE I

Following Methods WERE APPLIED IN THE EXPERIMENTS

\begin{tabular}{|l|l|}
\hline Method & Short Explanation \\
\hline No Weight & The solution given by (6). \\
Uniform Weight & Here (11) is minimized with the weights $w_{i j k}$ set to 1. \\
Edge-Induced & Here (11) is minimized with the weights $w_{i j k}$ set via the canny edge detetor. \\
Gradient-Induced & Here (11) is minimized with the weights $w_{i j k}$ set according to (13). \\
IHS Method & The IHS method, as described by (14). \\
Mean Cor. IHS & The mean corrected IHS method, as described by (14) and (16). \\
Brovey Method & The Brovey Method as described by (17). \\
\hline
\end{tabular}

TABLE II

Spectral Consistency of the IHS And BRovey Methods, Which Is MEAsured as THE CROSS CORRELATION BETWEEN THE LOW-RESOLUTION RGB AND APPROPRIATELY DOWN-SAMPLED Fused Images. All Other Applied Methods, CF., TABle I, ARE SPECTRALly CONSISTENT By Design, Which Has BEEN CONFIRMED EXPERIMENTALLY

\begin{tabular}{|c|c|c|}
\hline \multirow[t]{2}{*}{ Data set } & \multicolumn{2}{|c|}{ Spectral Consistency } \\
\hline & IHS & Brovey \\
\hline IKONOS & 0.9106 & 0.9089 \\
\hline QuickBird & 0.7932 & 0.8075 \\
\hline Meteosat & 0.9473 & 0.9380 \\
\hline
\end{tabular}

the panchromatic and low-resolution images is 16 or four by four.

3) Meteosat. A weather satellite image depicting Europe from the Meteosat satellite [26]. We use the R, G, and $\mathbf{B}$ bands of low resolution, and the ratio between the panchromatic and low-resolution images is nine or three by three.

Using these different data sets gives us an idea of how a given method will work on data sets with different contents. The reason that we did not use the fourth band in the IKONOS and QuickBird cases was for visualization purposes. We have, however, implemented and applied our fusion methods successfully to all four bands.

In evaluating the different methods, we were faced with the problem that we did not have any ground truth data, i.e., a high-resolution color image, to compare with. Due to this shortcoming, we have chosen two different strategies. First, we have applied the methods to the original data sets, computed the spectral consistency, see Table II, and visually evaluated the results, see Figs. 5-7. Second, we have down-sampled the original data to generate a new data set with ground truth, albeit with different intrinsic scale. The difference between this ground truth and the fused results was compared with different metrics, see Table III.

In the experiments, the $\gamma$ in (11) was set to five and one for the original and down-sampled data, ${ }^{8}$ respectively. In the Canny edge detector, which was used for generating the edgeinduced weights, the smoothing $\sigma$ was set to one and 0.05 for the original and down-sampled data, respectively. As for the gradient-induced weights, the parameters $\sigma$ and $\lambda$ were selected

${ }^{8}$ This was used only to generate the values in Table III.
TABLE III

RESUlt OF COMPARING THE FusEd RESUlt With THE GRound TRUth With the DOWN-SAMPled Data. HeRE, Bold DenOtes the Best

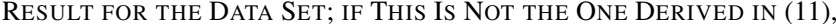
THE Best of These Is Denoted by ITALICS. SeE TABle I FOR A DESCRIPTION OF THE APPLIED METHODS

\begin{tabular}{|l|c|c|c|c|c|}
\hline & MSE & CC & WB & SSIM & Q4 \\
\hline IKONOS: & & & & & \\
No Weight & 0.0024 & 0.9120 & 0.6437 & 1.0000 & 0.6513 \\
Uniform Weight & 0.0029 & 0.8946 & 0.6133 & 1.0000 & 0.6171 \\
Edge-Induced & 0.0025 & 0.9094 & 0.6388 & 1.0000 & 0.6425 \\
Gradient-Induced & 0.0021 & 0.9244 & 0.6874 & 1.0000 & 0.6957 \\
IHS Method & 0.0026 & 0.8971 & 0.5523 & 0.9999 & 0.6386 \\
Mean Cor. IHS & $\mathbf{0 . 0 0 1 3}$ & $\mathbf{0 . 9 5 5 0}$ & $\mathbf{0 . 6 9 2 5}$ & 1.0000 & $\mathbf{0 . 7 2 0 1}$ \\
Brovey Method & 0.0248 & 0.8956 & 0.2053 & 0.9981 & 0.2422 \\
\hline QuickBird: & & & & & \\
No Weight & 0.0008 & 0.9132 & 0.7087 & 1.0000 & 0.7086 \\
Uniform Weight & 0.0008 & 0.9145 & 0.6844 & 1.0000 & 0.6809 \\
Edge-Induced & 0.0008 & 0.9195 & 0.7147 & 1.0000 & 0.7137 \\
Gradient-Induced & $\mathbf{0 . 0 0 0 7}$ & $\mathbf{0 . 9 2 1 3}$ & $\mathbf{0 . 7 2 2 7}$ & 1.0000 & $\mathbf{0 . 7 2 8 7}$ \\
IHS Method & 0.0132 & 0.6836 & 0.4470 & 0.9990 & 0.5217 \\
Mean Cor. IHS & 0.0008 & 0.8992 & 0.6666 & 1.0000 & 0.7003 \\
Brovey Method & 0.0138 & 0.6864 & 0.3543 & 0.9990 & 0.4056 \\
\hline Meteosat: & & & & & \\
No Weight & 0.0037 & 0.9491 & 0.7144 & 1.0000 & 0.6915 \\
Uniform Weight & 0.0033 & 0.9458 & 0.6851 & 1.0000 & 0.6836 \\
Edge-Induced & 0.0033 & 0.9474 & 0.6993 & 1.0000 & 0.6927 \\
Gradient-Induced & 0.0030 & 0.9545 & 0.7366 & 1.0000 & 0.7334 \\
IHS Method & 0.0114 & 0.9272 & 0.6277 & 0.9992 & 0.7293 \\
Mean Cor. IHS & $\mathbf{0 . 0 0 2 2}$ & $\mathbf{0 . 9 6 5 1}$ & $\mathbf{0 . 8 2 7 8}$ & 1.0000 & $\mathbf{0 . 8 3 8 4}$ \\
Brovey Method & 0.0713 & 0.9301 & 0.1581 & 0.9947 & 0.1614 \\
\hline & & & & & \\
\hline
\end{tabular}

as $(0.5,0.05)$ and $(0.5,0.007)$ for the original and down-sampled data, respectively. ${ }^{9}$

We have chosen five different metrics in evaluating the downsampled experiments, as reported in Table III. Many of the popular metrics are further developments of each other, which, in part, explain the strong correlation among the best performing

\footnotetext{
${ }^{9}$ The images are smoothed slightly with a Gaussian kernel before the gradient is calculated.
} 
methods. None or all of these metrics, however, encompass the ground truth of what a good fusion is; thus, visual inspection also needs to be taken into account. ${ }^{10}$ The used metrics, which are among the popular in the literature, are as follows (the boldface acronym indicate its entry in Table III).

1) MSE is the mean square error on all bands. It takes on values between zero and one with zero being the best value.

2) $\mathbf{C C}$ is the mean cross correlation between bands. It takes on values between zero and one with one being the best value.

3) WB is the mean Wang and Bovik measure between bands, cf., [27]. It takes on values between -1 and one with one being the best value.

4) SSIM is a quality metric which measures the degradation of structural information between two images. This metric combines three independent components of the images: Luminance, contrast, and structure, and the value one is only achieved if the compared images are identical. The lowest value is zero if the images show no similarity at all.

5) Q4 is an extension of the Wang and Bovik measure to multiple dimensions, giving a combined metric, cf., [28]. It takes on values between zero and one with one being the best value.

In Table III, most of the metrics (MSE, CC, WB, and Q4) give significant numerical differences for the individual methods which were applied on the different data sets. However, that is not the case for the SSIM metric, which gives similar results for all the methods that were applied on the different data sets. As a general observation, it is seen that the proposed methods work well. Specifically, the gradient-induced weight method should be noted, in that, it gives good visual results. Also, it performs well in the down-sample experiment. In comparison, the mean-corrected IHS method performs excellently in the down-sampled experiment but gives a too blocky result with the visual comparison. The Brovey and IHS methods give a very pleasing visual result but perform poorly with respect to the down-sampled experiment and are clearly not spectrally consistent.

Comparing the edge-induced and gradient-induced weighting schemes visually, it is seen that the edge-induced scheme gives an oversegmented image and the gradient-induced scheme visually gives the best results, although the latter image is a bit blurry. As mentioned in Section V, we have only presented two general approaches, and the search for the best weighting scheme is still ongoing. In this light, a mixture of the edge-induced and gradient-induced schemes seems well worth investigating.

The fact that the mean-corrected IHS method gives blocky images and the gradient-induced weight method gives a somewhat smoothed image is well in line with our current experience. This experience states that with the proposed method,

\footnotetext{
${ }^{10}$ Note, for example, that the mean-corrected IHS method performs best when the metrics are used for the comparison in our experiments, cf. Table III, but its visual quality leaves a lot to be desired. This motivates further investigation into image metrics, which is beyond the scope of this paper.
}

if spectral consistency is sought, either a blocking effect is endured, as discussed in Section VI, or a slightly blurred image is obtained because of the removal of the blockiness by smoothing. This can be explained by the panchromatic channel containing information that is not present in the RGB channels, cf., Fig. 1.

As a note for future work, neither our initial solution (also termed as the "no weight" solution) nor the mean-corrected IHS method uses a spatial prior, but both are spectrally consistent. Comparing the two, it is seen that the mean-corrected IHS method clearly outperforms the former in terms of the quality measures. This indicates that $\widehat{\mathbf{F}}_{i j}$ in (10) could possibly be replaced with the result of the mean-corrected IHS method. On the other hand, the IHS method is less related to the underlying imaging physics. Also, the IHS method, in some sense, works in the one-norm, see (14), instead of the induced two-norm. Using different norms also seems worth investigating, although these are also less tied to the imaging physics.

\section{CONCLUSION}

A framework has been presented for pixel-level fusion of satellite images, which is derived by considering the underlying imaging physics. This, among other characteristics, ensures spectral consistency by design. We have also argued that the satellite image fusion problem is inherently ill posed, and as such, our framework explicitly states how regularization should be performed to address that. Several schemes for regularization have been presented in the proposed framework. These schemes have been experimentally tested on several data sets. For all these data sets, the methods gave good performance. The schemes also held their ground when compared with other popular methods for satellite image fusion. Also, in this paper, the IHS method was revisited in order to gain additional insight into the implications of spectral consistency for image fusion methods. The results obtained with the proposed framework are promising, and further research on the framework is ongoing.

\section{APPENDIX \\ NUMERICAL SOLUTION}

It is noted that (11) is an LS system, a property which is retained after the reparameterization in (12) because it is just an affine mapping. In principal, the solution can be obtained by solving this LS system via the usual methods, e.g., the normal equations in [30]. However, this poses the problem that the dimension of the solution will often be very large. It can be computed as the number of high-resolution pixels times the number of bands. Therefore, LS methods become numerically prohibitive. As an example, the IKONOS data set requires 1.873.920 variables with just three bands. Thus, something else needs to be considered, e.g., exploiting the sparsity of the problem. It is noted that the LS property in (11) ensures that a nice and convex error surface is obtained with only one welldefined optimum. ${ }^{11}$

\footnotetext{
${ }^{11}$ Since the problem is regularized properly, the optimum is unique. Otherwise, the optimum would be a linear subspace.
} 
Our solution is solving (11) iteratively for all the highresolution pixels corresponding to a low-resolution pixel. Thus, we keep all other pixels constant in relation to the neighborhood term. The blocks were updated in a checkerboard fashion [31], [32]. The process was run until convergence, i.e., it was stopped when very small changes were observed in the solution.

\section{ACKNOWLEDGMENT}

The authors would like to thank A. Vésteinsson for spuring this work via his masters thesis [29] and the reviewers whose comments greatly improved this paper.

\section{REFERENCES}

[1] T. Carper, T. Lillesand, and R. Kiefer, "The use of intensity-huesaturation transformations for merging SPOT panchromatic and multispectral image data," Photogramm. Eng. Remote Sens., vol. 56, no. 4, pp. 459-467, 1990.

[2] P. Chavez and J. Bowell, "Comparison of the spectral information content of Landsat Thematic Mapper and SPOT for three different sites in the Phoenix, Arizona region," Photogramm. Eng. Remote Sens., vol. 54, no. 12 , pp. 1699-1708, 1988.

[3] K. Edwards and P. Davis, "The use of intensity-hue-saturation transformation for producing color shaded-relief images," Photogramm. Eng. Remote Sens., vol. 60, no. 11, pp. 1369-1373, 1994.

[4] T. Tu, P. Huang, C. Hung, and C. Chang, "A fast intensity-hue-saturation fusion technique with spectral adjustment for IKONOS imagery," IEEE Geosci. Remote Sens. Lett., vol. 1, no. 4, pp. 309-312, Oct. 2004.

[5] A. Gillespie, A. Kahle, and R. Walker, "Color enhancement of highly correlated images. II. Channel ratio and 'chromaticity' transformation techniques," Remote Sens. Environ., vol. 22, no. 3, pp. 343-365, Aug. 1987.

[6] P. Chavez, S. Sides, and J. Anderson, "Comparison of three different methods to merge multiresolution and multispectral data: Landsat TM and SPOT panchromatic," Photogramm. Eng. Remote Sens., vol. 57, no. 3, pp. 295-303, 1991.

[7] B. Aiazzi, L. Alparone, S. Baronti, and A. Garzelli, "Context-driven fusion of high spatial and spectral resolution images based on oversampled multiresolution analysis," IEEE Trans. Geosci. Remote Sens., vol. 40, no. 10 , pp. $2300-2312$, Oct. 2002

[8] J. Liu, "Smoothing filter-based intensity modulation: A spectral preserve image fusion technique for improving spatial details," Int. J. Remote Sens., vol. 21 , no. 18 , pp. 3461-3472, Dec. 2000.

[9] J. Nunez, X. Otazu, O. Fors, A. Prades, V. Pala, and R. Arbiol, "Multiresolution-based image fusion with additive wavelet decomposition," IEEE Trans. Geosci. Remote Sens., vol. 37, no. 3, pp. 1204-1211, May 1999.

[10] T. Ranchin and L. Wald, "Fusion of high spatial and spectral resolution images: The ARSIS concept and its implementation," Photogramm. Eng. Remote Sens., vol. 66, no. 1, pp. 49-61, 2000.

[11] M. J. Canty, Image Analysis, Classification and Change Detection in Remote Sensing: With Algorithms for ENVI/IDL. Boca Raton, FL: CRC Press, 2006.

[12] GeoEye, Geoeye Imaging Products Ikonos. [Online]. Available: $\mathrm{http} / / / \mathrm{www} . g e o e y e . c o m /$ products/imagery/ikonos/

[13] M. Pedersen, Functional Analysis in Applied Mathematics and Engineering. Boca Raton, FL: CRC Press, 1999.

[14] T. Anderson, An Introduction to Multivariate Statistical Analysis, 2nd ed. New York: Wiley, 1984.

[15] S. Li, Markov Random Field Modeling in Image Analysis, 2nd ed. New York: Springer-Verlag, 2007.

[16] G. Winkler, Image Analysis, Random Fields and Markov Chain Monte Carlo Methods: A Mathematical Introduction (Applications of Mathematics). New York: Springer-Verlag, 2003.

[17] R. Nishii, S. Kusanobu, and S. Tanaka, "Enhancement of low spatial resolution image based on high resolution-bands," IEEE Trans. Geosci. Remote Sens., vol. 34, no. 5, pp. 1151-1158, Sep. 1996.

[18] J. Canny, "A computational approach to edge detection," IEEE Trans. Pattern Anal. Mach. Intell., vol. PAMI-8, no. 6, pp. 679-698, Nov. 1986.

[19] L. Rudin, S. Osher, and E. Fatemi, "Nonlinear total variation based noise removal algorithms," Physica D, vol. 60, no. 1-4, pp. 259-268, Nov. 1992.
[20] J. Sveinsson, H. Aanæs, and J. Benediktsson, "Smoothing of fused spectral consistent satellite images with TV-based edge detection," in Proc. IGARSS, 2007, pp. 318-321.

[21] J. Weickert, Anisotropic Diffusion in Image Processing, ser. ECMI Series. Stuttgart, Germany: Teubner-Verlag, 1998

[22] P. Perona and J. Malik, "Scale-space and edge detection using anisotropic diffusion," IEEE Trans. Pattern Anal. Mach. Intell., vol. 12, no. 7, pp. 629-639, Jul. 1990.

[23] J. Zhou, D. Civco, and J. A. Silander, "A wavelet transform method to merge Landsat TM and SPOT panchromatic data," Int. J. Remote Sens., vol. 19, no. 4, pp. 743-757, Mar. 1998.

[24] M. Choi, "A new intensity-hue-saturation fusion approach to image fusion with a tradeoff parameter," IEEE Trans. Geosci. Remote Sens., vol. 44, no. 6, pp. 1672-1682, Jun. 2006.

[25] Quickbird Imagery Products. Product Guide, DigitalGlobe, Inc., Longmont, CO, 2006. Tech. Rep. 4.2.7. [Online]. Available: http://www.digitalglobe.com/downloads/QuickBird Imagery ProductsProduct Guide.pdf

[26] J. Schmetz, P. Pili, S. Tjemkes, D. Just, J. Kerkmann, S. Rota, and A. Ratier, "An introduction to Meteosat second generation (MSG)," Bull. Amer. Meteorol. Soc., vol. 83, no. 7, pp. 977-992, Jul. 2002.

[27] Z. Wang and A. C. Bovik, "A universal image quality index," IEEE Signal Process. Lett., vol. 9, no. 3, pp. 81-84, Mar. 2002.

[28] L. Alparone, S. Baronti, A. Garzelli, and F. Nencini, "A global quality measurement of pan-sharpened multispectral imagery," IEEE Trans. Geosci. Remote Sens. Lett., vol. 1, no. 4, pp. 313-317, Oct. 2004.

[29] A. Vesteinsson, J. Sveinsson, J. Benediktsson, and H. Aanæs, "Spectral consistent satellite image fusion: Using a high resolution panchromatic and low resolution multi-spectral images," in Proc. IGARSS, 2005, vol. 4 , pp. 2834-2837.

[30] Å. Björck, Numerical Methods for Least Squares Problems. Philadelphia, PA: SIAM, 1996.

[31] J. Besag, "On the statistical analysis of dirty pictures," J. R. Stat. Soc. B, vol. 48, no. 3, pp. 259-302, 1986.

[32] S. Geman and D. Geman, "Stochastic relaxation, Gibbs distributions, and Bayesian restoration of images," IEEE Trans. Pattern Anal. Mach. Intell., vol. PAMI-6, no. 6, pp. 721-741, Nov. 1984.

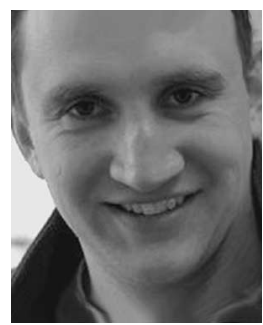

Henrik Aanæs received the M.Sc. degree in engineering and the Ph.D. degree from the Technical University of Denmark, Lyngby, in 2000 and 2003, respectively.

$\mathrm{He}$ is currently an Assistant Professor in computer vision and image analysis with the Department of Informatics and Mathematical Modelling, Technical University of Denmark. His main research interests are estimation of 3-D shape to form 2-D images, object recognition and tracking, statistical inference from images, data fusion, and robust statistics.

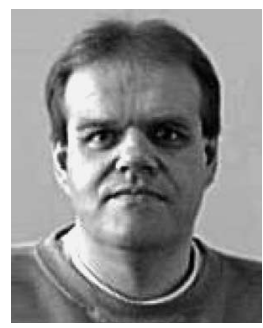

Johannes R. Sveinsson (S'86-M'90-SM'02) received the B.S. degree from the University of Iceland, Reykjavík, and the M.S. and Ph.D. degrees from Queen's University, Kingston, ON, Canada, all in electrical engineering.

$\mathrm{He}$ is currently an Associate Professor with the Department of Electrical and Computer Engineering, University of Iceland, where he was with the Laboratory of Information Technology and Signal Processing from 1981 to 1982 and, from November 1991 to 1998, the Engineering Research Institute and the Department of Electrical and Computer Engineering as a Senior Member of the research staff and a Lecturer, respectively. He was a Visiting Research Student with the Imperial College of Science and Technology, London, U.K., from 1985 to 1986. At Queen's University, he held teaching and research assistantships. His current research interests are in systems and signal theory.

Dr. Sveinsson received the Queen's Graduate Awards from Queen's University. 


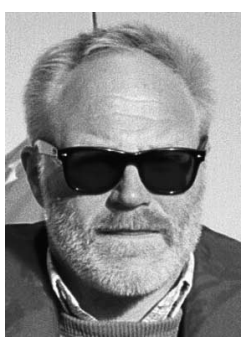

Allan Aasbjerg Nielsen received the M.Sc. and $\mathrm{Ph} . \mathrm{D}$. degrees from the Technical University of Denmark, Lyngby, in 1978 and 1994, respectively.

$\mathrm{He}$ is currently an Associate Professor with the National Space Institute, Technical University of Denmark, where he worked on energy conservation in housing with the Thermal Insulation Laboratory from 1978 to 1985 , with the section for image analysis in the Department of IMM from 1985 to 2001, as a Part-Time Acting Professor from 1995 to 2001, and with the section for geoinformatics in the Department of IMM from 2001 to 2006. He was with the Danish Defense Research Establishment from 1977 to 1978. Since 1985, he has been working on several national and international projects on the development, implementation, and application of statistical methods and remote sensing in mineral exploration, mapping, geology, agriculture, environmental monitoring, oceanography, geodesy, and security funded by industries, the European Union, the Danish International Development Agency, and the Danish National Research Councils. He is the Reviewer of several international journals and conferences.

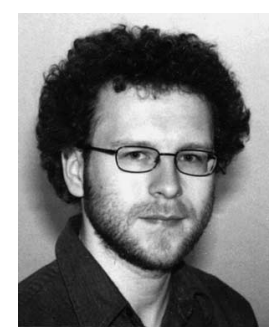

Thomas Bøvith received the M.S. degree in engineering from the Technical University of Denmark, Lyngby, in 2002, where he is currently working toward the Ph.D. degree in remote sensing in the National Space Institute, Technical University of Denmark, which is in collaboration with the Danish Meteorological Institute, Copenhagen.

His research interests include remote sensing of precipitation, data fusion, image classification, and scientific visualization.

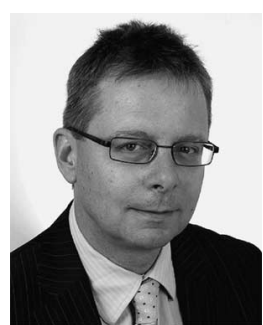

Jón Atli Benediktsson (S'84-M'90-SM'99-F'04) received the Cand.Sci. degree in electrical engineering from the University of Iceland, Reykjavík, in 1984 and the M.S.E.E. and Ph.D. degrees from Purdue University, West Lafayette, IN, in 1987 and 1990, respectively.

He is currently the Director of Academic Development and Innovation and a Professor with the Department of Electrical and Computer Engineering, University of Iceland. He has held visiting positions with the Department of Information and Communication Technology, University of Trento, Trento, Italy (2002-present); School of Computing and Information Systems, Kingston University, Kingston upon Thames, U.K. (1999-2004); the Joint Research Center of the European Commission, Ispra, Italy (1998); Technical University of Denmark, Lyngby (1998); and the School of Electrical and Computer Engineering, Purdue University (1995). $\mathrm{He}$ was a Fellow with the Australian Defense Force Academy, Canberra, A.C.T., in August 1997. From 1999 to 2004, he was the Chairman of the energy company Metan Ltd. His research interests are in remote sensing, pattern recognition, neural networks, image processing, and signal processing, and he has published extensively in those fields.

Dr. Benediktsson is the Editor of the IEEE TRANSACTIONS ON Geoscience and Remote Sensing (TGARS) and Associate Editor of the IEEE Geoscience and Remote Sensing LetTers. He was an Associate Editor of TGARS from 1999 to 2002. He coedited (with Prof. D. A. Landgrebe) a special issue on data fusion of TGARS (May 1999). In 2002, he was appointed as Vice President of Technical Activities in the Administrative Committee of the IEEE Geoscience and Remote Sensing Society (GRSS) and (with P. Gamba and G. G. Wilkinson) a special issue on urban remote sensing from satellite (October 2003). From 1996 to 1999, he was the Chairman of the GRSS Technical Committee on Data Fusion and was elected to the Administrative Committee of the GRSS for the term 2000 to 2002. In 2002, he was appointed as Vice President of Technical Activities of GRSS. He was the founding Chairman of the IEEE Iceland Section and served as its Chairman from 2000 to 2003 . He was the Chairman of the Quality Assurance Committee, University of Iceland (2006-present), the Chairman of the University's Science and Research Committee (1999-2005), a member of Iceland's Science and Technology Council (2003-2006), and a member of the Nordic Research Policy Council (2004). He was a member of the North Atlantic Treaty Organization Advisory Panel of the Physical and Engineering Science and Technology Subprogram (2002-2003). He received the Stevan J. Kristof Award from Purdue University in 1991 as an outstanding graduate student in remote sensing. In 1997, he was the recipient of the Icelandic Research Council's Outstanding Young Researcher Award, and in 2000, he was granted the IEEE Third Millennium Medal. In 2007, he received the Outstanding Service Award from the IEEE Geoscience and Remote Sensing Society. He is a member of Societas Scinetiarum Islandica and Tau Beta Pi. 